\title{
TCRP1 expression is associated with platinum sensitivity in human lung and ovarian cancer cells
}

\author{
XIAORONG LIU ${ }^{1,2}$, MEILING FENG $^{1}$, GUOPEI ZHENG ${ }^{1}$, YIXUE GU ${ }^{1}$, CHENGKUN WANG $^{1}$ and ZHIMIN HE ${ }^{1}$ \\ ${ }^{1}$ Affiliated Cancer Hospital and Cancer Research Institute, Guangzhou Medical University, Guangzhou, \\ Guangdong 510095; ${ }^{2}$ Shenzhen Engineering Laboratory for High-throughput Gene Sequencing of \\ Pathogens, Shenzhen Children's Hospital, Shenzhen, Guangdong 518038, P.R. China
}

Received December 12, 2014; Accepted December 22, 2015

DOI: $10.3892 / \mathrm{ol} .2016 .5534$

\begin{abstract}
Platinum-based drugs, including cisplatin (DDP) and oxaliplatin (L-OHP), are among the most potent chemotherapy drugs, and are widely utilized for the treatment of human lung and ovarian cancer. However, certain patients do not respond to platinum-based agents, and even those who initially benefit from the treatment will eventually exhibit resistance to these drugs. Although certain factors have been investigated for their potential to predict platinum resistance, more effective predictors for the improved management of patients with lung and ovarian cancer are required. Tongue cancer resistance-associated protein 1 (TCRP1) is a newly identified gene, which was cloned from a multi-drug resistant cell line of tongue cancer. Previous data has shown that TCRP1 is able to mediate DDP resistance in human oral squamous cell carcinoma cells. However, the contribution of TCRP1 to the resistance of platinum agents in human lung and ovarian cancer cells remains to be elucidated. Our previous study showed that TCRP1 expression levels in samples of lung and ovarian cancer were significantly increased compared with normal controls. In the present study, it was demonstrated that TCRP1 contributed to the resistance to DDP and L-OHP in human lung and ovarian cancer cells. Knockdown of TCRP1 resensitized the cells to the platinum-based agents. The present study identified a positive correlation between TCRP1 expression and primary resistance to DDP and L-OHP in lung cancer cells. In addition, it was observed that cells treated with nuclear factor (NF)- $\mathrm{B}$ inhibitor BAY 11-7082 displayed increased sensitivity to DDP and L-OHP. The results of the present study suggested that TCRP1 may be associated with resistance to DDP and L-OHP in lung and ovarian cancer cells,
\end{abstract}

Correspondence to: Professor Zhimin He, Affiliated Cancer Hospital and Cancer Research Institute, Guangzhou Medical University, 78 Hengzhigang Road, Yuexiu, Guangzhou, Guangdong 510095, P.R. China

E-mail: hezhimin2005@yahoo.com

Key words: tongue cancer resistance-associated protein 1, platinum sensitivity, lung cancer, ovarian cancer, nuclear factor- $\kappa \mathrm{B}$ and the Akt/NF- $\kappa \mathrm{B}$ signaling pathway may be involved in the functioning of TCRP1. These findings identify TCRP1 as a potential predictor of platinum resistance in the treatment of lung and ovarian cancer.

\section{Introduction}

Lung cancer is the most common and lethal type of cancer worldwide, and ovarian cancer is a leading cause of cancer-associated mortality in women $(1,2)$. In 2012 , the estimated global incidence rates for lung and ovarian cancer were 23.1 and 6.1 cases per 100,000 individuals, respectively, and the estimated global mortality rates were 19.7 and 3.7 mortalities per 100,000 individuals, respectively (3). Platinum-based antitumor drugs, including cisplatin (DDP) and oxaliplatin (L-OHP), are typical first-line agents that are used for the treatment of various types of cancer, including lung and ovarian cancer, as well as head and neck squamous cell carcinoma and lymphoma $(4,5)$. It is generally accepted that the efficacy of platinum-based therapy is modulated by drug uptake and efflux, cellular proliferation, DNA adduct formation and DNA repair (6). Platinum-based agents are able to form DNA intra- and inter-strand crosslinks, and DNA-protein complexes, which interfere with DNA synthesis, RNA transcription, the cell cycle and apoptosis $(7,8)$. L-OHP is a 1,2-diaminocyclohexane-containing platinum-based compound that is known to induce a reduced number of DNA double-strand breaks and possess decreased cytotoxicity compared with DDP $(9,10)$. However, certain patients do not respond to treatment with platinum-based agents, and even those who initially benefit from the treatment will eventually demonstrate resistance to these drugs $(11,12)$.

Clinically, drug resistance is divided into natural and acquired resistance (13). Acquired resistance to platinum develops during extended periods of treatment with platinum-based agents, while natural platinum resistance occurs in cells that have not previously been treated with platinum-based drugs (6). Therefore, predictive factors for improved management of lung and ovarian cancer patients with natural and acquired platinum resistance are urgently required. Although certain predictive markers, including excision repair cross-complementation group 1 (ERCC1) and Tau, have been investigated for their potential to predict 
platinum resistance in lung and ovarian cancer patients, more effective predictors require development and investigation $(12,14)$.

In a previous study, we cloned a novel gene from the Tca8113/pingyangmycin (Tca8113/PYM) tongue cancer multi-drug resistant cell line, which was termed tongue cancer resistance-associated protein 1 (TCRP1) (15). Notably, it was observed that TCRP1 contributed to DDP resistance in human oral squamous cell carcinoma (OSCC) cells (15-17). TCRP1 was reported to interact with Akt, and activation of the phosphoinositide 3-kinase/Akt/nuclear factor (NF)- $\kappa \mathrm{B}$ signaling pathway was involved in the functioning of TCRP1 in OSCC cells $(16,18)$. It was additionally demonstrated that TCRP1 has a significant role in the mediation of DDP resistance via increased cellular proliferation and decreased apoptosis (15). In another previous study, we reported that TCRP1 contributes to DDP resistance in A549 lung cancer cells, and that DNA repair protein polymerase $\beta(\mathrm{Pol} \beta)$ is involved in TCRP1-mediated DDP resistance (19). A previous study investigated the tissue-specific expression of TCRP1, and it was observed that TCRP1 expression levels in samples of lung and ovarian cancer were significantly increased compared with normal controls (data not published). However, the participation of TCRP1 in the resistance to platinum-based agents in human lung and ovarian cancer cells remains to be elucidated.

In the present study, the association between the expression of TCRP1 and the chemoresistance to DDP and L-OHP was analyzed in human lung and ovarian cancer cells. It was demonstrated that TCRP1 contributed to the resistance to DDP and L-OHP in lung and ovarian cancer cells, and knockdown of TCRP1 resensitized the cells to platinum-based agents. In addition, the present study identified that the $A k t / N F-\kappa B$ signaling pathway had a significant role in TCRP1-mediated platinum resistance. In summary, the results of the present study suggested that TCRP1 may be a potential predictor of platinum resistance in the treatment of lung and ovarian cancer.

\section{Materials and methods}

Reagents. Platinum-based agents DDP and L-OHP were purchased from Sigma-Aldrich (St. Louis, MO, USA). The NF- $\kappa$ B inhibitor BAY 11-7082 was obtained from EMD Millipore (Billerica, MA, USA).

Cell culture. The A549 human lung adenocarcinoma epithelial, A549DDP-resistant (A459/DDP), COC1 human ovarian cancer, COC1 DDP-resistant (COC1/DDP), MCF-7 human breast cancer, MCF-7 5-fluorouracil (5-Fu)-resistant (MCF7/5-Fu) and Tca8113 human OSCC cell lines were obtained from the China Center for Type Culture Collection (Wuhan, China). The PYM-induced Tca8113/PYM multidrug resistant cell line was previously established by Dr Guopei Zheng at the Cancer Research Institute of Central South University (Changsha, China) (20). The cells were maintained in RPMI 1640 medium (Invitrogen; Thermo Fisher Scientific, Inc., Waltham, MA, USA) containing $10 \%$ fetal calf serum (Invitrogen; Thermo Fisher Scientific, Inc.) at $37^{\circ} \mathrm{C}$ in a humidified atmosphere containing 5\% $\mathrm{CO}_{2}$. To maintain drug resistance, A549/DDP and COC1/DDP cells were routinely cultured with an additional $2 \mu \mathrm{g} / \mathrm{ml}$ DDP, and Tca8113/PYM cells were cultured with an additional $100 \mathrm{ng} / \mathrm{ml}$ PYM (Harbin Bolai Pharmaceutical Co., Ltd., Harbin, China) at $37^{\circ} \mathrm{C}$ in a humidified atmosphere containing $5 \% \mathrm{CO}_{2}$. The cells were additionally cultured in drug-free RPMI 1640 medium 1 week prior to starting the experiments at $37^{\circ} \mathrm{C}$ in a humidified atmosphere containing $5 \% \mathrm{CO}_{2}$. Histopathological subtypes of 8 human lung cancer cell lines, H1299, H1975, H460, H446, SK-MES-1, SPC-A-1, LTEP-a-2 and 95D, were obtained from the China Center for Type Culture Collection and cultured in RPMI 1640 medium containing $10 \%$ fetal calf serum at $37^{\circ} \mathrm{C}$ in a humidified atmosphere containing $5 \% \mathrm{CO}_{2}$.

Western blot analysis. Immunoblotting was performed as described previously (16). Briefly, whole cell lysates were prepared with radioimmunoprecipitation assay lysis buffer [50 mM Tris- $\mathrm{Cl}$ (pH 7.4), $150 \mathrm{mM} \mathrm{NaCl,} 1 \%$ Triton X-100, $1 \%$ sodium deoxycholate, $0.1 \%$ sodium dodecyl sulfate (SDS), $1 \mathrm{mM}$ sodium orthovanadate, $10 \mathrm{mM}$ sodium fluoride and $1 \%$ protease inhibitor cocktail; Thermo Fisher Scientific, Inc.]. The proteins were separated by $10 \%$ SDS-polyacrylamide gel electrophoresis and transferred onto a polyvinylidene difluoride membrane (Thermo Fisher Scientific, Inc.). The membrane was blocked with $5 \%$ v/w non-fat milk in $1 \mathrm{X}$ phosphate-buffered saline with Tween 20 to prevent non-specific binding, incubated with polyclonal rabbit anti-human TCRP1 (cat. no. sc-138365; 1:200; Santa Cruz Biotechnology, Inc., Dallas, TX, USA), monoclonal mouse anti-human $\beta$-actin (cat. no. sc-843; 1:500; Santa Cruz Biotechnology, Inc.), polyclonal rabbit anti-human p-Akt (cat. no. 9275; 1:1,000; Cell Signaling Technology, Inc., Danvers, MA, USA), monoclonal rabbit anti-human p-IкB (cat. no. 2859; 1:1,000; Cell Signaling Technology, Inc.) and polyclonal rabbit anti-human B-cell lymphoma (Bcl)-2 (cat. no. 2876; 1:1,000; Cell Signaling Technology, Inc.) primary antibodies and horseradish peroxidase-conjugated goat anti-rabbit (cat. no. 7074; 1:2,000; Cell Signaling Technology, Inc.) and horse anti-mouse (cat. no. 7076; 1:2,000; Cell Signaling Technology, Inc.) IgG secondary antibodies and detected with chemiluminescent substrate (ECL Western Blotting Detection kit; GE Healthcare Life Sciences, Chalfont, UK).

Reverse transcription-quantitative polymerase chain reaction (RT-qPCR). Cellular RNA was extracted from cells using TRIzol ${ }^{\circledR}$ reagent (Thermo Fisher Scientific, Inc.). The RNA was treated with 2 units DNase I (Thermo Fisher Scientific, Inc.). Complementary (c)DNA was synthesized using a First Strand cDNA Synthesis kit (Thermo Fisher Scientific, Inc.) according to the manufacturer's protocols. The expression levels of glyceraldehyde-3-phosphate dehydrogenase, which served as an internal control, and TCRP1 were verified by RT-qPCR using the Applied Biosystems 7500 Real-Time PCR System (Thermo Fisher Scientific, Inc.) with the SYBR Green Master Mix (Thermo Fisher Scientific, Inc.). PCR was performed under the following conditions: $95^{\circ} \mathrm{C}$ for $10 \mathrm{~min}$, followed by 40 cycles of $95^{\circ} \mathrm{C}$ for $15 \mathrm{sec}$ and $60^{\circ} \mathrm{C}$ for $1 \mathrm{~min}$. $\beta$-actin was used as an internal control. The fold change in relative expression of the target gene relative to $\beta$-actin was calculated as described previously (16). The following primers were designed using Primer3 (primer3.ut.ee/): TCRP1 forward, 5'-CCAATAGTC CCAGTTATGCTCCA-3' and reverse, 5'-TGCTTGGTAAGT 


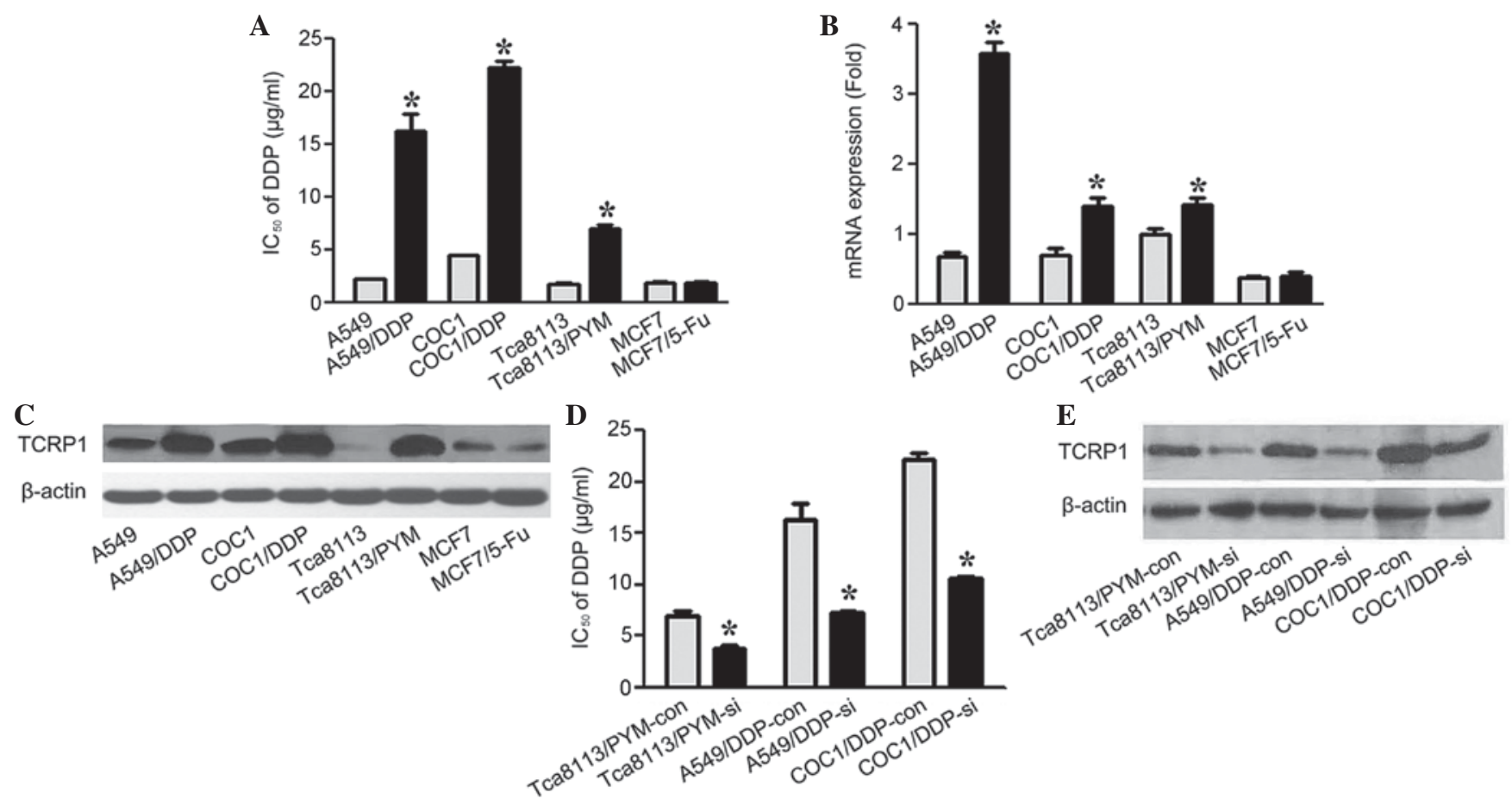

Figure 1. TCRP1 contributes to DDP resistance in lung and ovarian cancer cells with acquired platinum resistance. (A) $\mathrm{IC}_{50}$ values for DDP were analyzed. A549, COC1, Tca8113, MCF-7 and their relative drug-resistant cells were treated with various concentrations of DDP $(2.5,5.0,10.0,15.0,25.0 \mu \mathrm{g} / \mathrm{ml})$, and $\mathrm{IC}_{50}$ values were determined by dose-response curve. ${ }^{*} \mathrm{P}<0.05$ vs. control. (B) mRNA expression levels of TCRP1 in A549, COC1, Tca8113, MCF-7 and their drug resistant cells were detected by reverse transcription-quantitative polymerase chain reaction. ${ }^{*} \mathrm{P}<0.05$ vs. control. (C) Protein levels of TCRP1 in the cell lines were analyzed by western blotting. (D) TCRP1 siRNA or scramble control was transfected into Tca8113/PYM, A549/DDP and COC1/DDP cells for 48 h. The $\mathrm{IC}_{50}$ values for DDP in the indicated TCRP1 siRNA-treated cells and controls were investigated. "P $<0.05$ vs. control. (E) Protein expression levels of TCRP1 were detected by western blot analysis in the indicated TCRP1 siRNA-treated cells and controls. TCRP1, tongue cancer resistance-associated protein 1; DDP, cisplatin; $\mathrm{IC}_{50}$, half maximal inhibitory concentration; PYM, pingyangmycin; 5-Fu, fluorouracil; siRNA, small interfering RNA; si, siRNA-treated; con, control.

TCGGTTCTCG-3'; and $\beta$-actin forward, 5'-CTCACCATG GATGATGATATCGC-3' and reverse, 5'-AGGAATCCTTCT GACCCATGC-3'. Primers were synthesized by Invitrogen (Thermo Fisher Scientific, Inc.). Experiments were performed in triplicate.

Cytotoxicity assay. The cytotoxicity of DDP and L-OHP in various cell lines was determined by $3-(4,5-$ dimethylthiazol-2-yl)-5-(3-carboxymethoxyphenyl)-2-(4-sulfo phenyl)-2H-tetrazolium assay, as previously described (16). The absorbance was recorded at $490 \mathrm{~nm}$ using a Synergy 2 Multi-Mode plate reader (Bio-Tek Instruments, Inc., Winooski, VT, USA). Cell proliferation in the presence of DDP or L-OHP was detected based on the half maximal inhibitory concentration $\left(\mathrm{IC}_{50}\right)$ values determined by the dose-response curve.

$R N A$ interference. The cells were transiently transfected with the small interfering (si)RNA sequence against TCRP1 (5'-CCGAGAACCGAACUUACCA-3') and control oligonucleotides, as described previously (19). Lipofectamine ${ }^{\circledR}$ 2000 (Thermo Fisher Scientific, Inc.) was used to transfect the siRNA into the cells according to the manufacturer's protocols. The expression of TCRP1 was determined by western blot analysis.

Statistical analysis. Quantitative values are expressed as the mean \pm standard deviation. Spearman's rank correlation was calculated using SPSS version 13.0 (SPSS Inc., Chicago, IL, USA). Student's t-test was utilized to compare corresponding data. $\mathrm{P}<0.05$ was considered to indicate a statistically significant difference.

\section{Results}

TCRPI expression is associated with sensitivity to DDP in lung and ovarian cancer cells with acquired platinum resistance. To examine the role of TCRP1 in the acquired platinum resistance to DDP in lung and ovarian cancer cells, 4 cell lines and their drug-resistant equivalents were treated with various concentrations of DDP $(2.5,5.0,10.0,15.0,25.0 \mu \mathrm{g} / \mathrm{ml})$. $\mathrm{IC}_{50}$ values were determined by dose-response curve, and the mRNA and protein levels of TCRP1 were analyzed. As shown in Fig. 1A, the $\mathrm{IC}_{50}$ values of A549/DDP, COC1/DDP and Tca8113/PYM cells to DDP were significantly increased compared with parental cells $(\mathrm{P}=0.015, \mathrm{P}-0.021$ and $\mathrm{P}=0.039$, respectively). The results of RT-qPCR and western blot analysis revealed that A549/DDP, COC1/DDP and Tca8113/PYM cells expressed a significantly increased level of TCRP1 compared with the parental controls $(\mathrm{P}=0.016, \mathrm{P}=0.035$ and $\mathrm{P}=0.041$, respectively), in terms of the mRNA and protein levels (Fig. 1B and C). The present study demonstrated that the MCF7 and MCF7/5-Fu cells were sensitive to DDP treatment, and the expression levels of TCRP1 were relatively low in these two cell lines (Fig. 1A-C).

Subsequently, the effect of the siRNA knockdown of TCRP1 was detected in TCRP1-proficient cell lines, Tca8113/PYM, A549/DDP and COC1/DDP, and the TCRP1 siRNA or control was transfected into these three cell lines. 

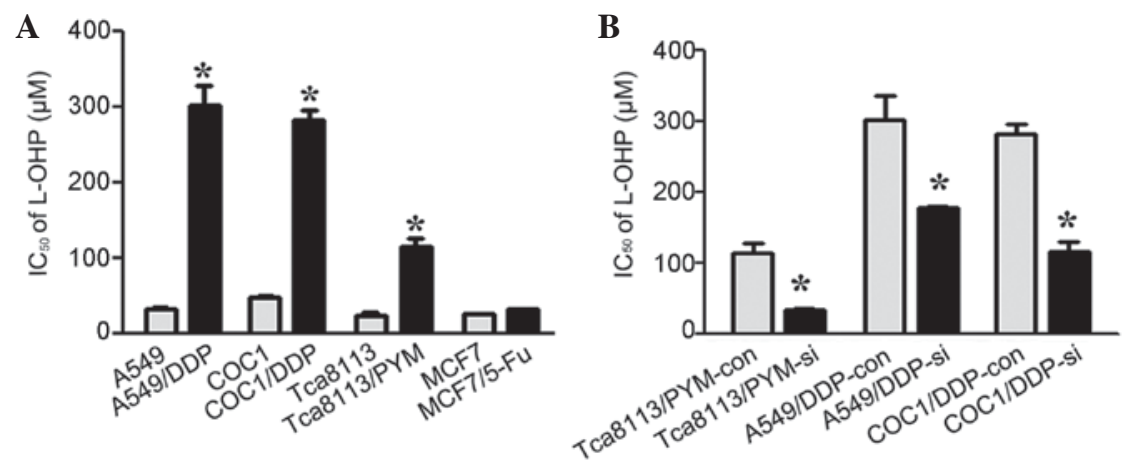

Figure 2. TCRP1 mediates resistance to L-OHP in lung and ovarian cancer cells with acquired platinum resistance. (A) A549, COC1, Tca8113, MCF-7 and their drug-resistant cells were treated with various concentrations of $\mathrm{L}-\mathrm{OHP}$, and $\mathrm{IC}_{50}$ values were determined by dose-response curve. ${ }^{*} \mathrm{P}<0.05$ vs. control.

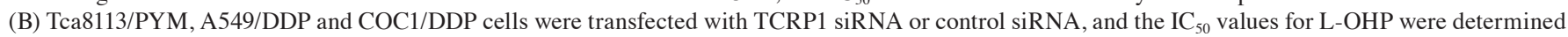
by dose-response curve. "P<0.05 vs. control. TCRP1, tongue cancer resistance-associated protein 1; L-OHP, oxaliplatin; siRNA, small interfering RNA; si, siRNA-treated; con, control; $\mathrm{IC}_{50}$, half maximal inhibitory concentration.

A

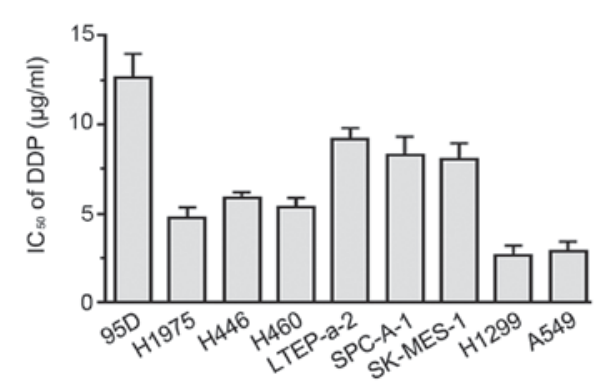

C

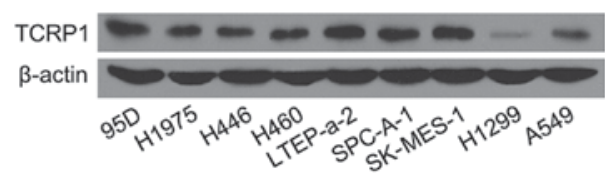

B

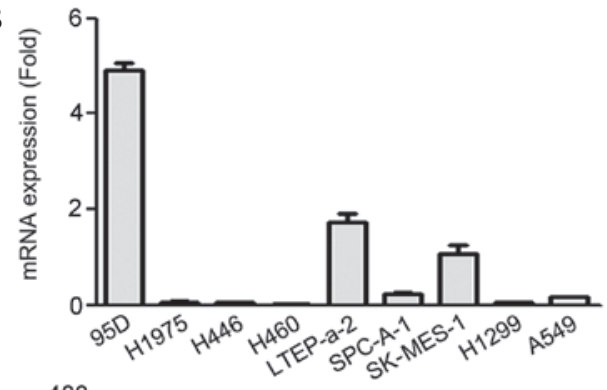

D

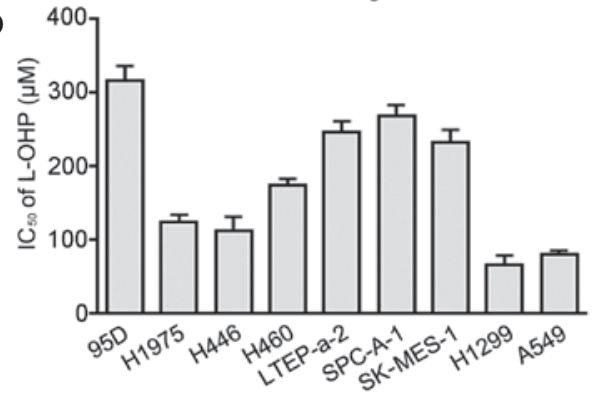

Figure 3. TCRP1 expression is associated with primary resistance to DDP and L-OHP in lung cancer cells. (A) A total of 9 lung cancer cell lines were treated with various concentrations of DDP, and $\mathrm{IC}_{50}$ values were determined by dose-response curve. (B) mRNA expression levels of TCRP1 in 9 lung cancer cell lines were detected by reverse transcription-quantitative polymerase chain reaction. (C) Protein levels of TCRP1 in the 9 lung cancer cell lines were detected by western blotting. (D) Various concentrations of L-OHP were added to the 9 lung cancer cell lines and the cells were cultured for 72 h. 3-(4,5-dimethylthiazol-2-yl)-5-(3-carboxymethoxyphenyl)-2-(4-sulfophenyl)-2H-tetrazolium assay was performed and the $\mathrm{IC}_{50}$ values for L-OHP were determined. TCRP1, tongue cancer resistance-associated protein 1 ; DDP, cisplatin; L-OHP, oxaliplatin; $\mathrm{IC}_{50}$, half maximal inhibitory concentration.

The cells were treated with the indicated concentrations of $\mathrm{DDP}$ and the dose-response curves were analyzed. The $\mathrm{IC}_{50}$ values for sensitivity to DDP following TCRP1-knockdown in Tca8113/PYM, A549/DDP and COC1/DDP cells were 1.8-, 2.3- and 2.1-fold lower compared with the controls, respectively (Fig. 1D). These results showed that the knockdown of TCRP1 increased the sensitivity to DDP in Tca8113/PYM, A549/DDP and COC1/DDP cells $(\mathrm{P}=0.037, \mathrm{P}=0.022$ and $\mathrm{P}=0.030$, respectively) (Fig. 1D and E). Therefore, this suggested that the expression of TCRP1 may be correlated with DDP resistance in lung and ovarian cancer cells with acquired platinum resistance.

TCRP1 mediates resistance to $L-O H P$ in lung and ovarian cancer cells with acquired platinum resistance. L-OHP is a third-generation platinum-based agent and has a markedly different spectrum of activity compared with DDP in vitro and in vivo (21). Clinical data has revealed that L-OHP has reduced toxicity and is therapeutically beneficial in the treatment of DDP-resistant tumors (10). To investigate whether TCRP1 mediated resistance to L-OHP in lung and ovarian cancer cells, A549, COC1, Tca8113, MCF-7 and their relative drug-resistant cells were incubated with various concentrations of $\mathrm{L}-\mathrm{OHP}$ and $\mathrm{IC}_{50}$ values were determined. Increased resistance to L-OHP was observed in TCRP1-proficient cells A549/DDP, COC1/DDP and Tca8113/PYM compared with their controls, and there was no significant change in sensitivity to L-OHP in the MCF-7 TCRP1-deficient and MCF-7/5-Fu drug-resistant cells $(\mathrm{P}=0.153)$ (Figs. $1 \mathrm{C}$ and $2 \mathrm{~A})$. To additionally identify the role of TCRP1 in L-OHP resistance, the expression of TCRP1 was knocked down in Tca8113/PYM, A549/DDP and 
A

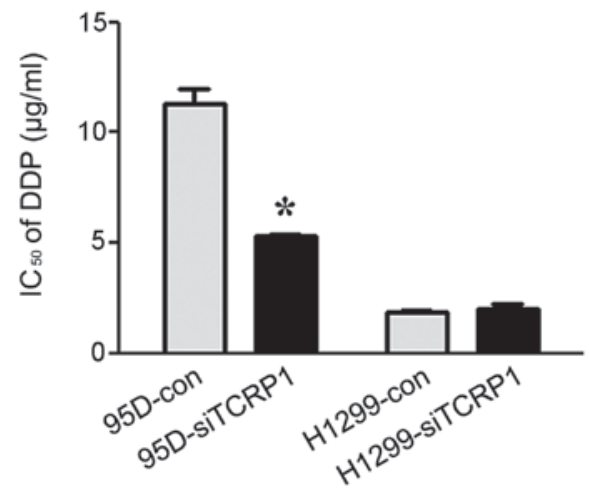

B

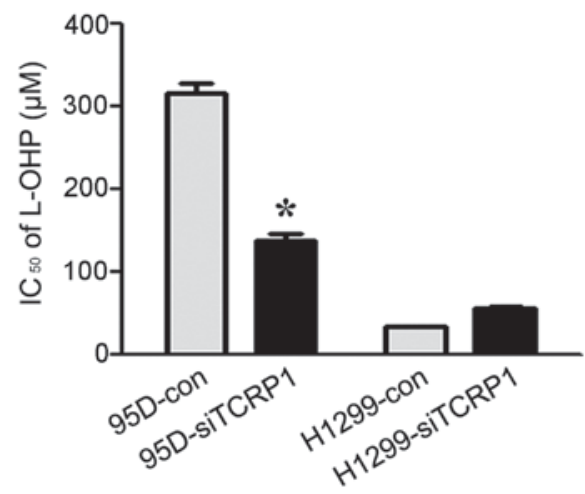

$\mathbf{C}$

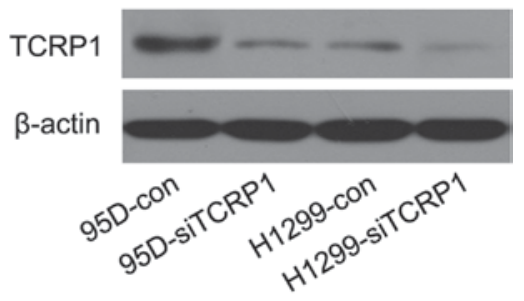

Figure 4. TCRP1 contributes to primary platinum resistance in lung cancer cells. (A) 95D and H1299 cells were transfected with TCRP1 siRNA or control. A total of $48 \mathrm{~h}$ after siRNA transfection, the cells were treated with various concentrations of DDP for $72 \mathrm{~h}$, and the $\mathrm{IC}_{50}$ values were determined by dose-response curve. ${ }^{*} \mathrm{P}<0.05$ vs. control. (B) $\mathrm{IC}_{50}$ values for L-OHP were determined by dose-response curve in $95 \mathrm{D}$ and H1299 cells treated with TCRP1-knockdown. ${ }^{*} \mathrm{P}<0.05$ vs. control. (C) Expression of TCRP1 was examined by western blotting analysis. TCRP1, tongue cancer resistance-associated protein 1; siRNA, small interfering RNA; DDP, cisplatin; con, control; $\mathrm{IC}_{50}$, half maximal inhibitory concentration.

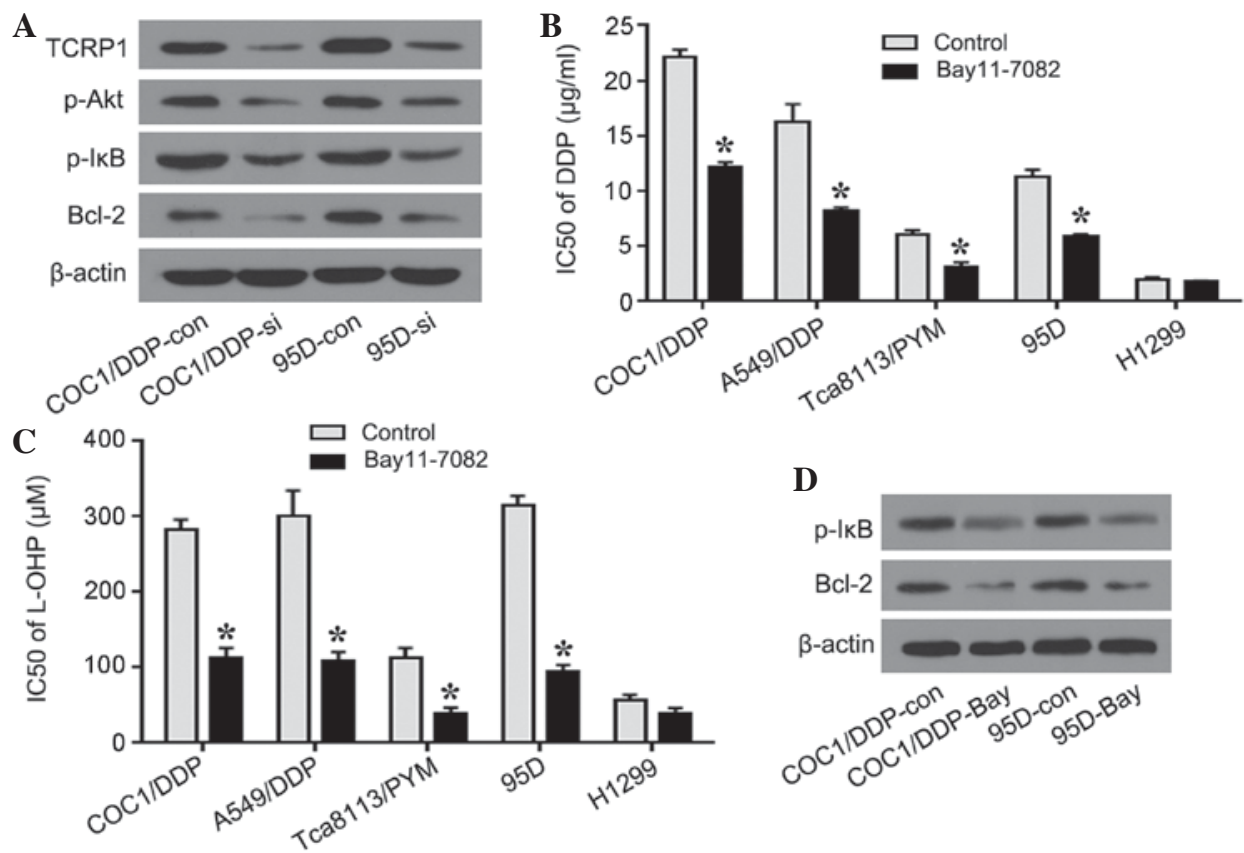

Figure 5. Akt/NF- $\mathrm{BB}$ signaling pathway is involved in TCRP1-mediated platinum resistance. (A) COC1/DDP and 95D cells were transfected with TCRP1 siRNA for $48 \mathrm{~h}$ and the expression of p-Akt, p-IкB $\alpha$ and Bcl-2 was investigated using western blotting analysis. (B) COC1/DDP, A549/DDP, Tca8113/PYM, 95D and H1299 cells were treated with NF-kB inhibitor BAY 11-7082 for $24 \mathrm{~h}$, and $\mathrm{IC}_{50}$ values for DDP were determined. "P<0.05 vs. control. (C) Cells were treated with BAY 11-7082 and the $\mathrm{IC}_{50}$ values for L-OHP were identified. ${ }^{*} \mathrm{P}<0.05$ vs. control. (D) COC1/DDP and 95D cells were treated with BAY 11-7082 for $24 \mathrm{~h}$ and $\mathrm{p}-\mathrm{I \kappa B} \alpha$ and $\mathrm{Bcl}-2$ were tested using western blotting analysis. NF, nuclear factor; TCRP1, tongue cancer resistance-associated protein 1; DDP, cisplatin; L-OHP, oxaliplatin; siRNA, small interfering RNA; si, siRNA-treated; con, control; p, phosphorylated; I $\mathrm{B} \alpha$, nuclear factor $\kappa \mathrm{B}$ inhibitor $\alpha$; Bcl-2, B-cell lymphoma 2; PYM, pingyangmycin; $\mathrm{IC}_{50}$, half maximal inhibitory concentration.

COC1/DDP cells and $\mathrm{IC}_{50}$ values for L-OHP were detected. As a result, increased sensitivity to L-OHP was detected in cells treated with TCRP1-knockdown, indicating that downregulation of TCRP1 may sensitize cells to L-OHP treatment (Fig. 2B). Taken together, these results suggested that TCRP1 may mediate resistance to L-OHP in lung and ovarian cancer cells with acquired platinum resistance.

A positive correlation exists between TCRPI expression and primary resistance to platinum in lung cancer cells. To assess the role of TCRP1 in the primary resistance to platinum-based agents, the $\mathrm{IC}_{50}$ values for DDP in 9 distinct histopathological subtypes of lung cancer cell lines were analyzed, and the TCRP1 expression was detected. The results of this analysis revealed that cell lines with higher expression levels of TCRP1 mRNA and protein demonstrated increased resistance to DDP (Fig. 3A-C). A positive correlation was observed between TCRP1 mRNA levels and DDP resistance in the 9 lung cancer cell lines $(r=0.78$; $\mathrm{P}=0.021$ ). L-OHP sensitivity was additionally detected in the aforementioned cell lines and it was observed that the $\mathrm{IC}_{50}$ values for L-OHP were increased in the cell lines with a 
higher expression level of TCRP1 (Fig. 3D). A positive correlation additionally existed between the mRNA expression of TCRP1 and L-OHP resistance in the 9 lung cancer cell lines $(\mathrm{r}=0.7 ; \mathrm{P}=0.036)$. Thus, the results indicated that TCRP1 expression may be associated with the primary resistance to DDP and L-OHP in lung cancer cells.

Primary platinum resistance in lung cancer cells may be reversed by TCRP1-knockdown. To further investigate the role of TCRP1 in the primary platinum resistance in lung cancer cells, the 95D and H1299 cell lines were selected as representatives of TCRP1-proficient and TCRP1-deficient lung cancer cells, respectively. The $\mathrm{IC}_{50}$ values for DDP and L-OHP in the 95D and H1299 cells were determined following TCRP1-knockdown. As shown in Fig. 4A-C, knockdown of TCRP1 resulted in the reversal of primary resistance to DDP and L-OHP in TCRP1-proficient 95D cells, while in TCRP1-deficient H1299 cells, no significant changes in platinum resistance were observed with or without TCRP1 knockdown $(\mathrm{P}=0.210$ and $\mathrm{P}=0.186$, respectively). This suggested that TCRP1 expression contributed to the primary resistance to platinum in lung cancer cells.

Akt/NF- $\kappa B$ signaling pathway is involved in TCRP1-mediated platinum resistance. Previously, we reported that the $\mathrm{Akt} / \mathrm{NF}-\kappa \mathrm{B}$ signaling pathway participated in TCRP1-associated DDP resistance in OSCC cells (16). To provide additional evidence that $\mathrm{Akt} / \mathrm{NF}-\kappa \mathrm{B}$ mediated the platinum resistance of TCRP1 in lung and ovarian cancer cells, the expression of phosphorylated (p)-Akt, p-NF- $\kappa \mathrm{B}$ inhibitor $\alpha(\mathrm{I} \kappa \mathrm{B} \alpha)$ and Bcl $\kappa-2$ was investigated using western blotting following the knockdown of TCRP1 in $\mathrm{COC1} / \mathrm{DDP}$ and 95D cells (Fig. 5A). As the activation of $\mathrm{NF}-\kappa \mathrm{B}$ requires removal of $\mathrm{I} \kappa \mathrm{B}$ by targeted phosphorylation and subsequent degradation, the expression of $\mathrm{p}-\mathrm{I} \kappa \mathrm{B} \alpha$ was assumed to represent the activity of $\mathrm{NF}-\kappa \mathrm{B}$ (22). Significant decreases in $\mathrm{p}$-Akt, $\mathrm{p}-\mathrm{I} \kappa \mathrm{B} \alpha$ and $\mathrm{Bcl}-2$ were observed in TCRP1-knockdown cells compared with the controls (Fig. 5A).

To additionally investigate the functional effect of $\mathrm{NF}-\kappa \mathrm{B}$ on platinum resistance, a platinum sensitivity assay was performed by treatment with $\mathrm{NF}-\kappa \mathrm{B}$ inhibitor BAY 11-7082. In acquired platinum resistance COC1/DDP, A549/DDP and Tca8113/PYM cell lines, the cells treated with BAY 11-7082 exhibited increased sensitivity to DDP, compared with control cells $(\mathrm{P}=0.043, \mathrm{P}=0.036$ and $\mathrm{P}=0.029$, respectively $)$. The $\mathrm{IC}_{50}$ value of $95 \mathrm{D}$ primary platinum resistance cells for DDP was significantly reduced following treatment with BAY 11-7082 compared with the controls $(\mathrm{P}=0.033)$ (Fig. 5B). Similarly, as shown in Fig. 5C, COC1/DDP, A549/DDP, Tca8113/PYM, 95D and H1299 cell lines exhibited increased sensitivity to L-OHP following treatment with BAY 11-7082, compared with the controls $(\mathrm{P}=0.030, \mathrm{P}=0.033, \mathrm{P}=0.041$ and $\mathrm{P}=0.037$, respectively). In addition, the expression of $\mathrm{p}-\mathrm{I} \kappa \mathrm{B} \alpha$ and $\mathrm{Bcl}-2$ was downregulated in the COC1/DDP and 95D cells following treatment with $\mathrm{NF}-\kappa \mathrm{B}$ inhibitor, suggesting that the knockdown of TCRP1 and the inhibition of NF- $\mathrm{NB}$ exhibited similar effects on the expression of $\mathrm{p}-\mathrm{I} \kappa \mathrm{B} \alpha$ and Bcl-2 (Fig. 5D). The results confirmed that TCRP1 was involved in the Akt/NF- $\mathrm{B}$ signaling pathway, and that the platinum resistance mediated by TCRP1 may function at least partially via $\mathrm{NF}-\kappa \mathrm{B}$ and $\mathrm{Bcl}-2$ in lung and ovarian cancer cells.

\section{Discussion}

The present study reports that TCRP1 has a significant role in platinum resistance in lung and ovarian cancer cells. The results of the present study demonstrated that TCRP1 expression is associated with sensitivity to DDP and L-OHP in lung and ovarian cancer cells with acquired platinum resistance, and that the knockdown of TCRP1 increases the sensitivity to DDP and L-OHP. It has additionally been identified that a positive correlation exists between TCRP1 expression and primary resistance to DDP and L-OHP in lung cancer cells. Furthermore, it was observed that the Akt/NF- $\kappa \mathrm{B}$ signaling pathway is involved in TCRP1-mediated platinum resistance. Therefore, the results of the present study suggest that TCRP1 may be a potential predictor for platinum resistance in the treatment of lung and ovarian cancer.

Although DDP and L-OHP are two of the most commonly used antitumor agents, resistance to platinum rapidly emerges following commencement of treatment (23). Primary resistance and acquired resistance are the two major forms of platinum resistance. A number of studies have focused on various mechanisms of platinum resistance (6). A serine/threonine protein kinase p70 ribosomal S6 kinase was reported to contribute to DDP resistance in lung cancer cells, and downregulation of this gene may circumvent DDP resistance (24). Yamano et al (23) compared the gene expression profiles between 3 DDP-sensitive OSCC cell lines and their relative DDP-resistant cell lines, and identified 5 novel genes that may have the potential for predicting the efficacy of DDP-based chemotherapy against OSCC. ERCC1 has been reported to be positively associated with the resistance to platinum-based chemotherapy in various tumors $(25,26)$. A microtubule-associated protein, Tau, has additionally been identified as a potential predictive marker in epithelial ovarian cancer patients treated with platinum and paclitaxel (12). However, more effective predictors of platinum resistance are required for the improvement of the diagnosis and treatment of lung and ovarian cancer.

Previously, a high expression level of TCRP1 has been shown to cause DDP resistance in OSCC cells (15). In the present study, it was observed that TCRP1 was highly expressed in acquired DDP-resistant lung (A549/DDP) and ovarian cancer (COC1/DDP) cells. As platinum-based agents are first-line clinical therapies in lung cancer patients, and $20 \%$ of tumors have been reported to exhibit primary resistance in clinical trials, the present study collected 8 distinct histopathological subtypes of human lung cancer cell lines to investigate the association between TCRP1 expression and primary platinum resistance $(27,28)$. The lung cancer cells that exhibited higher expression levels of TCRP1 showed increased resistance to DDP, and repression of TCRP1 expression contributed to the reversal of resistance to DDP and L-OHP. A positive correlation was observed between the expression of TCRP1 and the resistance to DDP in the investigated lung cancer cell lines. This suggested that TCRP1 may be a potential predictor of platinum resistance and its expression may assist with the selection of cancer patients who will benefit from platinum-based chemotherapy. Additional research is required to validate the results of the present study in a larger patient cohort. 
Akt is a serine-threonine protein kinase that is involved in cell growth, survival and proliferation (29). Activation of the Akt pathway has been identified to be one of the key mediators of platinum resistance (30). A previous study revealed that the expression of Akt and NF- $\kappa \mathrm{B}$ is reduced in Tca8113 cells treated with TCRP1 siRNA (18). In the present study, knockdown of TCRP1 expression contributed to increased sensitivity to platinum agents and downregulated expression of p-Akt, $\mathrm{p}-\mathrm{I} \kappa \mathrm{B} \alpha$ and $\mathrm{Bcl}-2$ in TCRP1 proficient cells, including COC1/DDP, A549/DDP, Tca8113/PYM and 95D cell lines. This suggested that the Akt/NF- $\mathrm{B} / \mathrm{Bcl}-2$ signaling pathway is involved in TCRP1-mediated platinum resistance in lung and ovarian cancer cells.

$\mathrm{NF}-\kappa \mathrm{B}$ is a transcriptional regulator and has significant roles in the cell cycle, apoptosis and stress responses (21). The activity of $\mathrm{NF}-\kappa \mathrm{B}$ is inhibited by $\mathrm{I} \kappa \mathrm{B} \alpha$ in the cytoplasm. Various intra- and extracellular stimuli lead to the phosphorylation of I $\mathrm{B} \alpha$, followed by subsequent polyubiquitination and degradation. Activated NF- $\kappa \mathrm{B}$ translocates into the nucleus and stimulates gene expression (31). In addition, constitutive $\mathrm{NF}-\kappa \mathrm{B}$ expression has been reported to exert an antiapoptotic effect on chemoresistance in cancer cells (32). NF- $\kappa \mathrm{B}$ binds the multidrug resistance protein 1 (MDR1) promoter and induces drug resistance via MDR1 expression (33). It is additionally known that NF- $\mathrm{NB}$ blocks the cytotoxicity induced by antitumor drugs via suppression of Bcl-2 phosphorylation (34). In the present study, knockdown of TCRP1 led to reversal of platinum resistance and the downregulation of $\mathrm{p}-\mathrm{I} \kappa \mathrm{B} \alpha$. Therefore, the present study subsequently aimed to elucidate the mechanisms underlying this reversal and investigate whether NF- $\mathrm{B}$ is involved in TCRP1-associated platinum resistance. The compound BAY 11-7082 selectively blocks tumor necrosis factor- $\alpha$-induced phosphorylation of $\mathrm{I} \kappa \mathrm{B} \alpha$ and inhibits the activation of NF- $\kappa \mathrm{B}$ (35). The present study observed that the sensitivity to DDP and L-OHP was markedly increased following treatment with BAY 11-7082 in platinum-resistant cancer cells. The results of the present study indicated that $\mathrm{NF}-\kappa \mathrm{B}$ may function as a downstream target protein involved in TCRP1-mediated platinum resistance. However, this observation requires further investigation in future studies.

Although previous research has focused on the functioning of TCRP1, the mechanisms of TCRP1 involvement in platinum resistance remain to be elucidated. One of our previous studies revealed that TCPR1 mediated DDP resistance via increasing the repair of DDP-induced DNA damage by preventing Pol $\beta$ degradation in lung cancer cells (19). In the present study, it was reported that TCRP1 expression is associated with the resistance to DDP and L-OHP in lung and ovarian cancer cells and that the Akt/NF- $\mathrm{BB}$ signaling pathway is involved in the functioning of TCRP1. The present study hypothesizes that there may be a functional link between the Akt/NF- $\mathrm{B}$ signaling pathway and Pol $\beta$ expression, and this interaction is currently under investigation.

In conclusion, the present study has shown that TCRP1 is associated with the resistance to DDP and L-OHP in lung and ovarian cancer cells, and that the Akt/NF- $\kappa \mathrm{B}$ signaling pathway is involved in the functioning of TCRP1. The results of the present study suggest that TCRP1 may be a potential predictor of platinum resistance, and a novel target for the prevention and reversal of platinum resistance in the treatment of lung and ovarian cancer.

\section{Acknowledgements}

The present study was supported by research grants from the Shenzhen Scientific and Technological Project (grant no. JCYJ20150403100317057), National Natural Science Foundation of China (grant nos. 30873088 and 81472184), the Natural Science Foundation of Guangdong Province (grant no. S2012010008995) and the Doctoral Fund of the Education Ministry of China (grant no. 20124423110003).

\section{References}

1. Liu C, Xu X and Zhou Y: Association between EGFR polymorphisms and the risk of lung cancer. Int J Clin Exp Pathol 8: 15245-15249, 2015.

2. Narod S: Can advanced-stage ovarian cancer be cured? Nat Rev Clin Oncol, Jan 20, 2016 (Epub ahead of print).

3. Ferlay J, Soerjomataram I, Ervik M, Dikshit R, Eser S, Mathers C, Rebelo M, Parkin DM, Forman D and Bray F: GLOBOCAN 2012 v1.0, Cancer Incidence and Mortality Worldwide: IARC CancerBase No. 11 [Internet]. International Agency for Research on Cancer. Lyon, France, 2013. http://globocan.iarc.fr. Accessed May 9, 2016.

4. Shen DW, Pouliot LM, Hall MD and Gottesman MM: Cisplatin resistance: A cellular self-defense mechanism resulting from multiple epigenetic and genetic changes. Pharmacol Rev 64: 706-721, 2012.

5. Macedo-Pérez EO, Morales-Oyarvide V, Mendoza-García VO, Dorantes-Gallareta Y, Flores-Estrada D and Arrieta O: Long progression-free survival with first-line paclitaxel plus platinum is associated with improved response and progression-free survival with second-line docetaxel in advanced non-small-cell lung cancer. Cancer Chemother Pharmacol 74: 681-690, 2014.

6. Siddik ZH: Cisplatin: Mode of cytotoxic action and molecular basis of resistance. Oncogene 22: 7265-7279, 2003.

7. Fikrova P, Stetina R, Hrnciarik M, Hrnciarikova D, Hronek M and Zadak Z: DNA crosslinks, DNA damage and repair in peripheral blood lymphocytes of non-small cell lung cancer patients treated with platinum derivatives. Oncol Rep 31: 391-396, 2014.

8. Galluzzi L, Senovilla L, Vitale I, Michels J, Martins I, Kepp O, Castedo M and Kroemer G: Molecular mechanisms of cisplatin resistance. Oncogene 31: 1869-1883, 2012.

9. Raymond E, Faivre S, Chaney S, Woynarowski J and Cvitkovic E: Cellular and molecular pharmacology of oxaliplatin. Mol Cancer Ther 1: 227-235, 2002.

10. Montagnani F, Turrisi G, Marinozzi C, Aliberti C and Fiorentini G: Effectiveness and safety of oxaliplatin compared to cisplatin for advanced, unresectable gastric cancer: A systematic review and meta-analysis. Gastric Cancer 14: 50-55, 2011.

11. Lowery WJ, Lowery AW, Barnett JC, Lopez-Acevedo M, Lee PS, Secord AA and Havrilesky L: Cost-effectiveness of early palliative care intervention in recurrent platinum-resistant ovarian cancer. Gynecol Oncol 130: 426-430, 2013.

12. Smoter M, Bodnar L, Grala B, Stec R, Zieniuk K, Kozlowski W and Szczylik C: Tau protein as a potential predictive marker in epithelial ovarian cancer patients treated with paclitaxel/platinum first-line chemotherapy. J Exp Clin Cancer Res 32: 25, 2013.

13. Zhao H, Bi T, Qu Z, Jiang J, Cui S and Wang Y: Expression of miR-224-5p is associated with the original cisplatin resistance of ovarian papillary serous carcinoma. Oncol Rep 32: 1003-1012, 2014.

14. Kuhlmann JD, Wimberger P, Bankfalvi A, Keller T, Schöler S, Aktas B, Buderath P, Hauch S, Otterbach F, Kimmig R and Kasimir-Bauer S: ERCC1-positive circulating tumor cells in the blood of ovarian cancer patients as a predictive biomarker for platinum resistance. Clin Chem 60: 1282-1289, 2014.

15. Gu Y, Fan S, Xiong Y, Peng B, Zheng G, Yu Y, Ouyang Y and He Z: Cloning and functional characterization of TCRP1, a novel gene mediating resistance to cisplatin in an oral squamous cell carcinoma cell line. FEBS Lett 585: 881-887, 2011.

16. Peng B, Gu Y, Xiong Y, Zheng G and He Z: Microarray-assisted pathway analysis identifies MT1X \& NFкB as mediators of TCRP1-associated resistance to cisplatin in oral squamous cell carcinoma. PLoS One 7: e51413, 2012. 
17. Peng B, Yi S, Gu Y, Zheng G and He Z: Purification and biochemical characterization of a novel protein-tongue cancer chemotherapy resistance-associated protein1 (TCRP1). Protein Expr Purif 82: 360-367, 2012.

18. Gu Y, Fan S, Liu B, Zheng G, Yu Y, Ouyang Y and He Z: TCRP1 promotes radioresistance of oral squamous cell carcinoma cells via Akt signal pathway. Mol Cell Biochem 357: 107-113, 2011.

19. Liu X, Wang C, Gu Y, Zhang Z, Zheng G and He Z: TCRP contributes to cisplatin resistance by preventing Pol $\beta$ degradation in lung cancer cells. Mol Cell Biochem 398: 175-183, 2015.

20. Zheng G, Zhou M, Ou X, Peng B, Yu Y, Kong F, Ouyang Y and He Z: Identification of carbonic anhydrase 9 as a contributor to pingyangmycin-induced drug resistance in human tongue cancer cells. FEBS J 277: 4506-4518, 2010.

21. Voland C, Bord A, Péleraux A, Pénarier G, Carrière D, Galiègue $\mathrm{S}$, Cvitkovic $\mathrm{E}$, Jbilo $\mathrm{O}$ and Casellas $\mathrm{P}$ : Repression of cell cycle-related proteins by oxaliplatin but not cisplatin in human colon cancer cells. Mol Cancer Ther 5: 2149-2157, 2006.

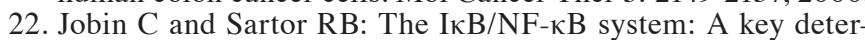
minant of mucosal inflammation and protection. Am J Physiol Cell Physiol 278: C451-C462, 2000.

23. Yamano Y, Uzawa K, Saito K, Nakashima D, Kasamatsu A, Koike H, Kouzu Y, Shinozuka K, Nakatani K, Negoro K, et al: Identification of cisplatin-resistance related genes in head and neck squamous cell carcinoma. Int J Cancer 126: 437-449, 2010.

24. Dhar R and Basu A: Constitutive activation of p70 S6 kinase is associated with intrinsic resistance to cisplatin. Int J Oncol 32: 1133-1137, 2008

25. Lee SH, Noh KB, Lee JS, Lee EJ, Min KH, Hur GY, Lee SH, Lee SY, Kim JH, Lee SY, et al: Thymidylate synthase and ERCC1 as predictive markers in patients with pulmonary adenocarcinoma treated with pemetrexed and cisplatin. Lung Cancer 81: 102-108, 2013.

26. Steffensen KD, Waldstrøm M and Jakobsen A: The relationship of platinum resistance and ERCC1 protein expression in epithelial ovarian cancer. Int J Gynecol Cancer 19: 820-825, 2009.
27. Florea AM and Büsselberg D: Cisplatin as an anti-tumor drug: Cellular mechanisms of activity, drug resistance and induced side effects. Cancers (Basel) 3: 1351-1371, 2011.

28. Haslehurst AM, Koti M, Dharsee M, Nuin P, Evans K, Geraci J, Childs T, Chen J, Li J, Weberpals J, et al: EMT transcription factors snail and slug directly contribute to cisplatin resistance in ovarian cancer. BMC Cancer 12: 91, 2012.

29. Cao H, Zhu K, Qiu L, Li S, Niu H, Hao M, Yang S, Zhao Z, Lai Y, Anderson JL, et al: Critical role of AKT protein in myeloma-induced osteoclast formation and osteolysis. J Biol Chem 288: 30399-30410, 2013.

30. Stronach EA, Chen M, Maginn EN, Agarwal R, Mills GB, Wasan $\mathrm{H}$ and Gabra $\mathrm{H}$ : DNA-PK mediates AKT activation and apoptosis inhibition in clinically acquired platinum resistance. Neoplasia 13: 1069-1080, 2011

31. $\mathrm{Oh} \mathrm{H}$ and Ghosh S: NF-кB: Roles and regulation in different CD4(+) T-cell subsets. Immunol Rev 252: 41-51, 2013.

32. Arlt A, Gehrz A, Müerköster S, Vorndamm J, Kruse ML, Fölsch UR and Schäfer H: Role of $N F-\kappa B$ and Akt/PI3K in the resistance of pancreatic carcinoma cell lines against gemcitabine-induced cell death. Oncogene 22: 3243-3251, 2003.

33. Bentires-Alj M, Barbu V, Fillet M, Chariot A, Relic B, Jacobs N, Gielen J, Merville MP and Bours V: NF- $\kappa$ B transcription factor induces drug resistance through MDR1 expression in cancer cells. Oncogene 22: 90-97, 2003.

34. Pham CG, Bubici C, Zazzeroni F, Knabb JR, Papa S, Kuntzen C and Franzoso G: Upregulation of Twist-1 by NF- $\kappa$ B blocks cytotoxicity induced by chemotherapeutic drugs. Mol Cell Biol 27: 3920-3935, 2007.

35. Sun H, Wu Y, Fu D, Liu Y and Huang C: SIRT6 regulates osteogenic differentiation of rat bone marrow mesenchymal stem cells partially via suppressing the nuclear factor- $\kappa \mathrm{B}$ signaling pathway. Stem Cells 32: 1943-1955, 2014. 